\title{
EL PAPEL DE LA COMUNICACIÓN EN LA BÚSQUEDA DE LA IDENTIDAD EN LA ETAPA ADOLESCENTE. ADOLESCENCIA: IDENTIDAD Y COMUNICACIÓN
}

\section{THE ROLE OF COMMUNICATION IN THE SEARCH FOR IDENTITY IN THE ADOLESCENT STAGE. ADOLESCENCE: IDENTITY AND COMMUNICATION}

Javier Sierra Sánchez: Profesor de Comunicación e Información Audiovisual I. Universitat Abat Oliba CEU jsierras@uao.es

Pedro Octavio Reyes Enríquez: Director de las Licenciaturas en Ciencias de la Comunicación e Historia del Arte de la Universidad Cristóbal Colón. Coordinador del proyecto de investigación-acción "Programa diocesano de lucha contra las adicciones"

preyes@aix.ver.ucc.mx

Miguel Ángel Córdoba Zamudio: Jefe del Instituto Calasanz de Ciencias de la Educación en América. Catedrático de Psicología de la Universidad Cristóbal Colón macz@aix.ver.ucc.mx

\section{CURRÍCULUM VITAE}

-Profesor de Teoría de la Comunicación de la Universidad San Jorge de Zaragoza (España). Doctor en Ciencias de la Información por la Universidad Complutense de Madrid (España). Licenciado en Comunicación Audiovisual y Periodismo por la Universidad Complutense de Madrid. Experto en Relaciones Públicas Internacionales por la Universidad Complutense de Madrid (España). Experto en 
Protocolo y ceremonial del estado e Internacional por la Universidad de Oviedo (España) y la Escuela Diplomática de Madrid (España). Ha impartido numerosas ponencias en congresos y seminarios nacionales e internacionales. Ha participado en varios proyectos de investigación financiados por el Ministerio de Educación y Ciencia, MEC y por la Universidad Complutense de Madrid (España). Ha sido becario FPI de la Universidad Complutense de Madrid. Es miembro fundador de la revista científica Icono 14.

-Sociólogo, con especialidad en docencia y maestría en educación superior. Ha realizado varias investigaciones de corte cualitativo sobre el fenómeno de los medios de comunicación y su relación con la salud cuyos resultados ha publicado en varias revistas impresas y electrónicas. Ha impartido cursos y talleres en varias universidades del país, también ha sido ponente en congresos nacionales e internacionales. Colabora en medios impresos y electrónicos del estado de Veracruz (México). Fue responsable de la academia de investigación de la licenciatura en Ciencias de la Comunicación de la Universidad Cristóbal Colón (México) en donde actualmente es catedrático y director de la misma.

-Psicólogo por la Universidad Cristóbal Colón (México) y Maestría en Educación Superior, Docente Investigador adscrito a la Licenciatura en Psicología de la Universidad Cristóbal Colón (México), ha publicado en varias revistas impresas y electrónicas temas del campo de psicología y salud. Actualmente es Director del Instituto Calasanz de Ciencias de la Educación - Educación Continua de la Universidad Cristóbal Colón (México) e Investigador Asociado de la Red Iberoamericana en Familia y Medios de Comunicación (FAMECOM) en México. 


\section{RESUMEN}

En el presente trabajo se analiza un fenómeno que puede presentarse durante la adolescencia, al cual se le ha denominado "Una adolescencia sin otredad", en donde por momentos el adolescente parece visualizarse en una dinámica y feroz lucha por su autodeterminación, la búsqueda de su identidad, de su autonomía e individualidad. Durante este proceso parece experimentar un aislamiento, una separación de sus contexto primario de formación (familia), para refugiarse principalmente en el grupo de amigos, sin embargo, nuestros adolescentes manifiestan una sensación de vacío, de soledad, de un sin sentido de vida, y enfrentan su tiempo de crecimiento y desarrollo sin la presencia de los otros (padres, amigos, etc.) e inclusive el desear no estar con ellos, experimentando el sentirse poco apoyados por su entorno. En este espacio de vida los medios de comunicación ocupan un lugar especial, pero además se derivan otros comportamientos los cuales se describen en este trabajo, que es resultado de la investigación que se hizo en la zona conurbada Veracruz-Boca del Río. Este estudio se basó en el instrumento metodológico elaborado por el proyecto "Medios y Salud: la voz de los adolescentes". ${ }^{1}$

\footnotetext{
${ }^{1}$ Se basó en la propuesta metodológica elaborada por Rafael Obregón y Cols. (2001) para la OPS proyecto COMSALUD, a la cual se le hicieron adaptaciones contextuales. Las técnicas de recopilación de la información fueron la encuesta y el grupo focal. Se aplicaron 1200 cuestionarios a población de secundaria, bachillerato y nivel técnico, así como 300 a adolescentes no escolarizados, la muestra se determinó por segmentos de población a partir del nivel socioeconómico de los padres. Se realizaron 24 grupos focales, los cuales se aplicaron a población de nivel secundaria, bachillerato, nivel técnico y adolescentes no escolarizados, se dividieron por género. Al cuestionario original elaborado por el Proyecto COMSALUD (Obregón y Cols., 2001) se le agregaron cuarenta reactivos más, así mismo se le hicieron algunas adaptaciones semánticas, igualmente se tomó como base las categorías de los grupos focales elaboradas para este estudio. Agradecemos el apoyo metodológico de la Red Iberoamericana en Familia y Medios de Comunicación (FAMECO)
} 


\title{
PALABRAS CLAVE
}

Comunicación - Adolescencia - Otredad - Salud

\begin{abstract}
In the following paper we will analyze a phenomenon which may occur during adolescence, and which we will name "An adolescence without the presence of others", where an adolescent, from time to time, tends to visualize oneself in a fierce and dynamic struggle to obtain one's selfsufficiency, identity, autonomy and individuality. During this process one seems to experimence isolation, or rather, separation from it's primary contexts. In this difficult moments the adolescents are under a lot of pressure, which, in turn, it is manifested in loneliness, a life without a cause and a growth of oneself which is not supported by any members of an adolescent's surrounding (parents, friends). A wish of loneliness and helplessness might be created within oneself. In this particular period of life, the means of communication strive to take a special place. Apart from that, there are other components and/or factors which will be described in this paper done with the sole purpose for the co - urban zone of Veracruz and Boca del Rio. It is based on the methodological factors elaborated which were conducted in a project entitled: "Media and Health: the voice of the Adolescents".
\end{abstract}

\section{KEY WORDS}

Communication - Adolescence - Otherness - Health

\section{ÍNDICE}


1. Introducción

2. Las amistades y el nuevo círculo social no responde a sus expectativas

3. Ruptura con los padres

4. Deserción social del adolescente

5. Medios

6. Concluyendo

7. Fuentes de consulta

\section{TEXTO}

\section{Introducción}

A lo largo de dos años de estudio y convivencia con los adolescentes de la zona conurbada Veracruz-Boca del Río, hemos observado fenómenos importantes como el incremento en el consumo de sustancias adictivas, principalmente alcohol y tabaco; un interés menor en las actividades escolares cotidianas, una mayor disposición a pasar más tiempo en actividades de ocio, mayor dependencia a nuevas opciones de comunicación: Internet, celulares, chat, correo electrónico. Sin embargo, hay un factor que resalta por encima de todos los anteriores y en estos momentos del estudio consideramos que se le ha dado poca atención, además, que sus consecuencias aún 
no se tienen claras, pero que a muchos jóvenes abarca y es el hecho de que estén viviendo una adolescencia sin otredad; caracterizada por:

El adolescente generalmente, en casa, se encuentra solo, o bien, bajo la responsabilidad de un hermano mayor o familiar que no se responsabiliza ni interactúa con él, los padres están pocas horas presentes.

Tiene una madre con una doble jornada o a ésta le resulta más atractivo distraerse con los medios de comunicación o con cualquier otra actividad, lo que impide compartir tiempo con el adolescente.

Tiene un padre ausente, sea porque éste trabaja (es un padre proveedor) o porque no vive con él, pocas veces tiene un padre educador.

La educación y la formación de valores no se realiza en casa, sino en otros espacios ajenos al primer núcleo familiar. Es en el ámbito de los amigos, los tíos o tías, vecinos o el personal doméstico en donde él tiene su aprendizaje axiológico.

Sus afectos se vierten más hacia sus amigos, novi@, ciberamigos y/o cibernovi@s, con los cuales desarrolla una implicación emocional que a veces le puede generar daño por las condiciones en que se da.

Tienen padres permisivos, los cuales no establecen claridad en las reglas familiares.

Se aísla de su entorno para dedicar tiempo a los medios de comunicación.

Los "otros" casi no tienen presencia en su vida. Están con él a su alrededor pero no responden a sus necesidades 
Se siente poco apoyado por su entorno, considera que la familia y los amigos no lo aprecian lo suficiente ni le brindan el tiempo y espacio que necesita.

Los anteriores puntos no son una constante en todos los jóvenes, pero varios de estos aspectos sí se presentan en un sector de la población en estudio en alguna etapa de su vida. Es importante aclarar la concepción que se le da en este trabajo a la otredad la cual de manera simple es entendida como los otros, el grupo de afuera (de acuerdo a Fossaert, en Gall, 2004) sin embargo es un concepto polisémico, usado en diversos campos como la filosofía, la poesía, la psicología, la antropología y la sociología entre otros y en cada uno de ellos los distintos autores le dan diferentes significados. En este trabajo se parte del principio de otredad en donde el adolescente considera al otro como parte externa de él y que es ajeno a su actuar y los valora a partir de sus propios principios y valores (Montero, 2001) y se manifiesta con un deseo de convivir con los miembros de su entorno (Flores, 1999). A continuación se explica el fenómeno observado.

volver al primer apartado volver al principio del artículo volver al principio

\section{Las amistades y el nuevo círculo social no responde a sus expectativas}

Tradicionalmente se considera que en la adolescencia el sujeto amplía su círculo social y la relación familiar comienza a debilitarse (Aguirre Baztán, 1996), lo último es cierto, el individuo inicia un proceso de independencia respecto a sus padres, lo cual genera ajustes en la personalidad $\mathrm{y}$, por supuesto, problemáticas, incluso algunas veces conductas de riesgo; pero en cuanto a su ámbito social, no siempre sucede, veamos, si bien llega a considerar que sus amigos siempre estarán con él, 
siempre lo apoyarán, estarán a su lado en todo momento; sobreestima la dimensión de la amistad, como lo relataron en los grupos focales realizados:

Pienso que mis amigos siempre estarán conmigo, la amistad es para toda la vida, y creo que yo al igual que ellos daría todo lo que pudiera si tuvieran algún problema. Claro, hablo de mis amigos cercanos, de ésos que lo ayudan a uno en todo y siempre que pueden.

Gisela, 15 años, $2^{\circ}$ de secundaria.

Sin embargo, en este estudio se observa que a pesar de que el círculo social se va ampliando, no responde a las expectativas del propio adolescente, en donde él espera más afecto y complicidad:

En la secundaria tengo igual de amigos que en la primaria, casi son los mismos, conozco más gente eso sí, voy a más lados, ya no estoy pegado tanto a mamá, pero a mis compañeros no les tengo confianza, ellos no me ayudan en todo, al contrario, a veces se la pasan molestándome, yo hago lo mismo también, los "friego" cada vez que puedo, pero amigos amigos en quien confiar pocos... nada más Juan, y hasta eso, luego anda de marica contando cosas que le he dicho.

Saúl, 14 años, $2^{\circ}$ de secundaria.

Cada ruptura con los amigos implica un nuevo ajuste, que muchas veces puede orillar a estados de depresión. Lo mismo sucede cuando hay noviazgo adolescente, se idealiza demasiado la relación, se considera que la pareja estará siempre al lado y de manera incondicional. Por otro lado, en esas relaciones de noviazgo muchas veces se da la violencia, en donde ni siquiera los mismos protagonistas de la relación lo perciben, generalmente el proceso se observa como normal, ya que es "común que el novio sea celoso" (Jovita, 17 años, bachillerato), lo cual permite cualquier tipo de reclamo; como señala Aldame, (2003) posteriormente se puede convertir en un 
insulto, sometimiento, golpes y violencia sexual. En la investigación el 14 \% de las entrevistadas declararon haber sufrido algún tipo de violencia y en los grupos de discusión se observó que ésta no siempre era percibida como tal. El dato anterior contrasta con otros obtenidos por el INEGI y el Instituto Nacional de la Mujer, quienes señalan que un $21.5 \%$ de las mujeres sufre de violencia por parte de su novio, esposo o compañero. Actitudes y comportamientos que atentan contra la integridad física o emocional no se consideran violentos; las mujeres y los hombres que los viven señalan que son parte del proceso de la relación, la violencia dentro del amor se encuentra naturalizada.

Cuando la violencia excede lo que el sujeto puede soportar, o bien, éste se percata de que la situación lo rebasa y no sabe qué hacer, la relación no siempre se rompe, continúa, lo cual genera soledad; se puede tener acompañante pero no compañía, por lo tanto, la afectividad que desea gozar todo sujeto no se ve satisfecha. En estos casos, el individuo entra en una dinámica contradictoria que va del amor al miedo y viceversa.

El anhelo de encontrar una pareja llega a veces a ser el máximo deseo de los y las adolescentes, no es solamente cubrir la afectividad, sino también un elemento que le da estatus ante los demás miembros de su grupo. El hecho de tener pareja implica dentro de su imaginario estar sobrevalorado por los miembros de su grupo. A la pregunta expresa de si les gustaría tener novi@, en la encuesta realizada, de los adolescentes de 11 a 14 años que declararon no tener, un 67\% contestó que sí; entre los de 15 a 18, un 83\% contestó de manera afirmativa, dentro de la población escolarizada; y en la población no escolarizada los valores aumentan, en el primer grupo de edad el $81 \%$ señaló que sí y en el segundo un $96 \%$. 
Existen trabajos (Guadarrama, Valero y Brito, 2004 ) que señalan las relaciones de pareja en los adolescentes es una conducta de riesgo cuando éstos llegan a la experimentación de dicha conducta sin una formación previa, sin idea del sentido, significado y fines del noviazgo, lo cual por desgracia es lo más común en nuestro entorno, la información sobre las relaciones de pareja la obtiene de lo que proyecta la televisión a través de telenovelas juveniles cargadas de escenas de celos, de lucha y violencia justificada por el amor, traiciones, seducciones, permisividad para múltiples parejas y encuentros sexuales, sin mencionar lo que las revistas juveniles ofertan "10 tips para ligar al hombre de tu vida", "descubre si tu hombre ideal te es fiel” , “¿es tu pareja ideal?”, sumado a lo que amig@s suelen enseñarle, condiciones como éstas colocan al adolescente actual en lo que denominamos condiciones de riesgo por todo lo que puede generar el asumir y reproducir estos modelos mediáticos de conducta: embarazos no deseados, aborto, ETS, violencia psíquica, física y sexual, depresión; sin embargo, es una etapa que posibilita la maduración del sujeto en diversas áreas psicoafectivas del individuo. Sin duda, el grupo de edad que en nuestro país más riesgo tiene de generar embarazos no deseados, abortos e ITS, es la población adolescente. De acuerdo con ONUSIDA, el 50\% de las nuevas infecciones se produjeron en jóvenes del grupo de edad de 10 a 24 años (Ramos Cavazos y Cantú Martínez, 2003)

Las características en las amistades que buscan los jóvenes, de acuerdo con Hurlock (1995), son muy distintas a las del niño y el adulto. Se buscan aquellos sujetos en quienes "Se pueda confiar y de quien sea posible depender, alguien con quien se pueda conversar y cuyos intereses sean similares a los propios" (1995: 133), incluso la apariencia es importante, debe ser afín a los intereses del sujeto, no idéntica pero que en ella se vea reflejada el contexto cultural de la mayoría de los adolescentes. 
Hurlock (1995) señala que idealizan a los amigos, les atribuyen aspectos que realmente no tienen, hay un enamoramiento del otro; esta situación muy pronto provoca rupturas, por el hecho de no poder profundizar en las relaciones de amistad. El adolescente considera que el amigo debe dar todo por él, como él mismo lo haría; debe ser su confidente quien guarda todos sus secretos, siempre disponible para él, incluso, que sustituya el papel de la familia. Los amigos posibilitan la independencia emocional del círculo familiar, se busca que éstos den estabilidad afectiva. En la sociedad veracruzana, para los jóvenes es difícil encontrar amigos con esos atributos, y es que como señalan autores como Conger (1999:70): “Los adolescentes desean que sus amigos sean leales, dignos de confianza y constituyan una fuente de apoyo en cualquier crisis emocional", sin embargo, tal tipo ideal no es fácil de encontrar:

Amigos de la flota, tengo muchos, pero no se puede confiar en ellos, quisiera... es que mire, no son flota, para el desmadre todos son buenos, pero ni uno es chitón (callado)... cuando realmente hay broncas serias, cada quien se rasca con sus propias uñas, a veces se siente uno "remal" pero así es... quisiera tener amigos, amigos, pero todos son bien cabrones...

Ramiro, 15 años, secundaria

El adolescente desea que su amigo sea un ser perfecto, alguien incondicional y que le satisfaga sus necesidades afectivas. Esto también suelen trasladarlo al o la que será o es su pareja. En la medida que el adolescente está más tiempo desvinculado de su familia, esto se refuerza, busca que aquellos elementos que no le son satisfechos en el hogar le sean dados en otros lugares.

Para el adolescente las amistades son algo fundamental, en parte, éstas orientan su vida y la van conformando. Si uno lee autores de los 80 (Mckinney, Fitzgerald, Strommen, entre otros), o anteriores, que escriben sobre la adolescencia, éstos 
concentran capítulos importantes sobre las formas de amistad y de cómo éstas, muchas veces, llegan hasta determinar la vida del sujeto; sin embargo, hoy se puede observar cómo las relaciones de amistad se van modificando, están mediadas por distintos procesos como son los medios de comunicación, en donde las nuevas tecnologías obligan o permiten otras formas de establecer lazos de afecto, en donde no se puede todavía establecer un juicio y decir que son peores o mejores que antes, ni hacer una evaluación para considerar que son perjudiciales o benéficos. Las generaciones que utilizan las nuevas tecnologías para relacionarse, como el chat, el correo electrónico, los MSN por medio del teléfono celular, el propio teléfono celular, entre otras, son muy recientes; en Veracruz la llegada de la Internet se da en 1996 y los primeros servicio de chat públicos surgen en 1998, su uso se vuelve más cotidiano entre los jóvenes a partir del 2000, que es cuando el servicio aparece por distintos puntos de la ciudad. Por lo tanto, es muy pronto para hacer una evaluación clara sobre el comportamiento de las relaciones afectivas mediadas por las nuevas tecnologías, aunque a nivel nacional ya hay algunos trabajos que hacen un balance hasta cierto punto positivo.

Un importante vínculo con los amigos son los medios de comunicación y las nuevas tecnologías, las opiniones sobre comunicarse a través de estos medios son claras, para ellos es más práctico. Ahora, el aspecto que se encuentra dividido es el de establecer relaciones afectivas exclusivamente por estos medios $\mathrm{O}$ predominantemente, algunos jóvenes dicen que es "malo", que "no tiene chiste", otros que es "buen principio", o bien, una forma de "prolongar una amistad"; el mismo joven que dice que "están mal las relaciones por Internet", reconoce que depende del caso y que conoce casos de personas que se han conocido por medio de Internet y después se han casado, algunos aceptan haber tenido noviazgos exclusivamente por este medio y que inclusive se llegaron a enamorar. 
Los medios de comunicación y las nuevas tecnologías son un espacio en donde los jóvenes establecen relaciones, buscan amistades, prolongan relaciones, buscan modelos ideales de amor y sexualidad que les sirven como referentes en su vida cotidiana.

\section{Ruptura con los padres}

Dentro del estudio se observó adolescentes que tratan de independizarse completamente de la autoridad de los padres, situación que regularmente no logran, pero siguen anhelando. Lo cual va a marcar nuevas formas de relacionarse con ellos, que generalmente tienden a ser más impersonales, en donde el lazo afectivo se busca que sea menos fuerte. Aguirre Baztán (1998) señala que el adolescente "huye" de su niñez, al considerar que ésta lo ata y limita a normas y comportamientos que no le permiten independizarse y encontrar su identidad.

Sus modelos de identificación en la mayoría de los casos ya no son sus padres (o bien, quienes lo criaron: abuelos, tíos, tutor, etc.), esto le genera conflictos al interior de su ámbito familiar; incluso llegan a desdeñar todo lo que apreciaban en su niñez, incluyendo a su figura materna y materna, de los cuales hasta se llega a burlar, en gran parte esto como una señal de independencia y de libertad como lo señala Aguirre Baztán (1998). En lo general, los jóvenes tienden a desarrollar un sentimiento de divergencia hacia el mundo y entre menos edad tengan y socialmente se encuentren menos favorecidos, su postura tiende a ser más radical (Brito, 2002).

La verdad mis padres no son lo que yo quisiera, cometen muchos errores, mi padre no gana mucho y mi madre es floja, sí trabaja, pero en las tardes se la pasa viendo la televisión, y los domingos se pone a platicar con sus comadres...

Saúl, 16 años, $3^{\circ}$ de secundaria. 
El adolescente se distancia de los "otros" que un día contribuyeron a formar su identidad y los niega como parte de su modelo de vida; va buscando una nueva identidad, lo cual conlleva que se distancie de los lazos afectivos de su niñez. Sustituye el papel de los padres como figuras de identidad por otras que pueden ser desde amigos de sus padres, familiares cercanos, líderes de barrio, hasta deportistas o estrellas del mundo del espectáculo reales o ficticios. Esto último muchas veces no lo llega a aceptar, pero se refleja en su comportamiento, forma de vestir y de hablar.

Dentro del estudio realizado a la población adolescente, se exploró la dinámica de interacción entre los miembros familiares, así como la conformación de las familias en las que los adolescentes se encuentran integrados. Un punto de indagación se ubicó con respecto a si habían tenido conflictos (Peleas, discusiones, etc) en su casa en los últimos seis meses, el 54\% contestó que sí, y cuando se les preguntó con quién, éstos fueron los resultados. 2

Cuadro 1: Personas con las que han tenido conflictos los adolescentes en los últimos seis meses

Persona con la que se tuvo el conflicto

Papá
$\%$

34 
Mamá 36

$\begin{array}{ll}\text { Hermanos } & 12\end{array}$

Tíos 3

Abuelos $\quad 2$

Otros familiares 4

Otras personas 4

Sin conflicto $\quad 46$

Fuente: Encuesta Adolescencia sin otredad, 2005.

Del total de la población adolescente, un 35\% reportó que ha tenido conflictos con papá y mamá, un 10\% con todos los miembros de la familia nuclear (papá, mamá y hermanos) y solamente un $2 \%$ con todos los que viven dentro de la casa.

El adolescente desea actuar libremente, imponiendo sus propios objetivos y hacer lo que le agrada, lo cual también muchas veces le genera rupturas con el ámbito familiar; dependiendo del tipo de padres que tenga, si éstos son permisivos o indiferentes, el problema tal vez sea menor, pero si son autoritarios definitivamente el conflicto será mayor. En el siguiente cuadro(2), elaborado a partir del guión de entrevista de Otero-López (1997) se muestra el grado de permisibilidad que tienen los adolescentes:

Cuadro 2: Autonomía del adolescente de acuerdo con el tipo de actividad que realizan 


\begin{tabular}{|c|c|c|c|c|}
\hline Tener novio & 24.1 & 13.9 & 49.1 & 9.3 \\
\hline Fumar & 78.7 & 2.8 & 15.7 & 1.9 \\
\hline Salir con amigos & 2.8 & 88.9 & 7.4 & 0.0 \\
\hline Beber alcohol & 75.0 & 9.3 & 11.1 & 3.7 \\
\hline Vestir como quieras & s 2.8 & 11.1 & 84.3 & 0.9 \\
\hline Llegar tarde & 26.9 & 65.7 & 2.8 & 3.7 \\
\hline Tatuarse & 77.8 & 13.9 & 5.6 & 1.9 \\
\hline Tener relaciones sex & xuales 57.4 & 6.5 & 22.2 & 13.0 \\
\hline Teñirse el cabello & 39.8 & 36.1 & 17.6 & 5.6 \\
\hline
\end{tabular}

Fuente: Encuesta Adolescencia sin otredad, 2005

Los jóvenes entonces dejan de identificarse plenamente con los valores familiares y sociales (Aguirre Baztán, 1998), en algunos casos quieren romper con ellos. Muchas veces actúan precisamente en contra de éstos con la intención de reafirmarse, desarrollando conductas de riesgo pudiendo ser éstas hasta delictivas. Desde luego los padres ante este tipo de conducta antes, tienden a cuestionar las actitudes de independencia y de cierto aislamiento de los hijos y les cuesta trabajo comprender los cambios del niño a adolescente, pero en lo general, no hay un solo patrón de respuesta. Si bien están conscientes de que su hijo está sufriendo cambios y que dejará de actuar como lo hacía antes, sin embargo se les hace difícil sobrellevar esa situación.

Todo lo anterior le genera soledad y estados de estrés al adolescente, que pueden derivar en complicaciones serias, desde luego que esto último no siempre sucede, a veces es sólo un ajuste que supera. El adolescente, ante esa sensación de estar solo, se vuelve más vulnerable y sensible, lo cual genera que sus sentimientos no siempre los 
comparta por el temor de salir lastimado, aislándose un poco más de los demás, separándose de los otros.

Es conveniente mencionar que cuando los padres son totalmente permisivos, su actuar no se traduce en hijos que se sientan comprendidos, muchas veces es contraproducente, ya que para autores como Moreno Kena (2003), Velasco Fernández (2001 y 2003) e instituciones como el CONADIC y el CENATI es un factor de riesgo que puede ser una causante de alguna adicción a las drogas. Sin duda, el adolescente demanda libertad, pero también es indispensable que se le pongan límites. En los grupos de discusión esto salía a la luz:

Hay profes que de plano no están interesados en tenernos quietos, dicen que a ellos les vale, llegan, medio dan la clase, para cubrir el tiempo, se ponen a leer y se van... $\mathrm{Y}$ es que como todos los compañeros gritan, avientan cosas, entonces nadie pone atención y no aprendemos, eso no se vale, deben actuar, como otros maestros que ésos desde que entran ponen quietos a todos. En la casa pasa lo mismo, mire, cuando a mi hermano lo dejan que haga lo que quiera, hace de las suyas, así era mi mamá con él, pero lo mandaron con mi abuela, y hasta trabaja y estudia, pero ella se lo trae bien "cortito", si los padres no se ponen duros, cada quien hace lo que se le antoja...

Laura, 17 años, estudiante de $1^{\circ}$ de bachillerato.

Algunos adolescentes también mencionaron que a veces sus propios padres son completamente permisivos y en otras ocasiones son muy estrictos, incluso para la misma actividad, lo cual genera confusión, además de que visualizan las normas familiares como un capricho paterno. Lo cierto es que la relación con los padres en la adolescencia se modifica, esto genera un desajuste en el hijo y en el padre; el primero no sabe cómo actuar y el segundo no sabe muchas veces cómo tratarlo. 
2- Podían contestar con más de una opción.

\section{Deserción social del adolescente}

Brito (2002) considera que la tendencia social actual es que en lugar de insertar al joven a la sociedad, hay una "deserción social", ya que los adolescentes están desertando de la escuela, la familia y demás instituciones tradicionales

Hurlock (1995) considera que existen "adolescentes sociales", los cuales actúan conforme a las normas, tienen un desempeño esperado por el grupo y están satisfechos por actuar de acuerdo con lo que los demás esperan de él, pero también existen "adolescente asociales", quienes no pueden adaptarse a la norma del contexto debido a falta de competencias sociales; para este autor también existe el “adolescente antisocial", el cual se encuentra consciente de lo que el grupo espera de él, pero por circunstancias personales actúa en contra. Efectivamente, hay adolescentes que no pueden integrarse a su grupo, que no están capacitados para la convivencia, debido a que desde niños permanecen en poco contacto con los padres, lo cual les genera dificultades para convivir en comunidad de acuerdo con Hurlock (1995); es indispensable que tengan un guía para aprender formas de comportamiento social y aprendan a relacionarse, éste no puede convertirse de manera automática en un adolescente socializado; en lo general, el hecho de que de niño tenga poco contacto con los miembros de su entorno familiar y poca satisfacción en su infancia genera que tenga escasa motivación para las actividades sociales. Entonces, hay adolescentes que no están capacitados y otros que no muestran interés para incorporarse a una vida normada por reglas que facilitan la convivencia. Las 
instituciones no están preparadas para trabajar con este tipo de adolescentes, ni las escuelas ni los maestros tienen generalmente los conocimientos para poder tratarlos, la familia a veces ni siquiera sabe cómo sobrellevarlos, por lo tanto, muchas veces cuando éste empieza a separarse de la vida escolar o familiar, para todos aparentemente es "más cómodo".

La deserción escolar en nivel secundaria es alta, como lo ha reconocido el propio Secretario de Educación Pública (Madrigal, 2004), de acuerdo con datos de la CEPAL (2002), la deserción escolar en México es del 35\% en zonas urbanas (población de nuestro interés), por encima de varios países de América Latina como Chile (14\%), Perú (16\%), República Dominicana (19\%), Argentina 23\%, Brasil 23\%, Colombia 24\%, Panamá 25\% Costa Rica 30\%, Ecuador 28\%, El Salvador 30\%, Nicaragua 34\%, Paraguay 32\% y Uruguay 32\%. En la zona conurbada Veracruz-Boca del Río, para el 2004, era aproximadamente del $29 \%$ por debajo de la media nacional, lo cual es un indicador de lo dicho en el párrafo anterior, el contexto social no tiene la capacidad de mantener al adolescente estudiando, lo cual va ligado con condiciones económicas, un sistema educativo, el ambiente familiar y el desarrollo del individuo principalmente.

Hay sectores de adolescentes que se sienten excluidos de los distintos contextos y otros que se autoexcluyen, generando sus propios grupos, lo cual no indica que no convivan en espacios colectivos, lo hacen pero bajo su propia identidad, diferenciándose desde el vestir de la mayoría. La deserción social de los adolescentes es aprovechada por las organizaciones de delincuentes, los cuales los arropan y permiten que actúen con absoluta libertad:

El "chito" era mi amigo, vendía droga y lo detuvieron, salió libre porque es menor de edad; él lo hacía no solamente por dinero, también porque se drogaba, y si no lo 
hacía, su flota lo "pendejeaba". Eran unos chavos que se ponían en la esquina, mayores que él, entonces se la pasaba con ellos toda la tarde, en su casa estaba solo, su mamá trabaja y su papá vive en otra parte, me parece que con otra "ñora", supuestamente una vecina lo vigilaba, y siempre su mamá le decía que ya no fuera con esos cuates, pero pues le valía lo que le dijera porque casi no estaba con él... entonces esos güeyes eran como su familia... al principio me burlaba de él, pero ya después ni me pelaba, mi mamá me dice que no me junte con él, ya que es un delincuente y es que lo meten a las casas a robar...

Marcial, 15 años, adolescente no escolarizado.

En las bandas de roba autos, aproximadamente el 20 y 30\% son adolescentes y un 10\% es de catorce años o menos (Herrera, 2004). La delincuencia organizada está dándoles "espacios" y brindándoles ciertos satisfactores que las instituciones como la escuela y la familia no son capaces de darles. Por otro lado, les permiten sacar el resentimiento social que traen acumulado a través de actos vandálicos. Los adolescentes tradicionalmente se sienten sin poder social, lo cual les produce frustración y desesperanza (Ramos y otros, 2002), pero cuando se incorporan a bandas delictivas consideran que ellos son capaces de ejercer poder y control. En este estudio detectamos muy pocos adolescentes que trabajan en bandas, sin embargo, en la zona conurbada Veracruz-Boca del Río han destacado varias -como la de Los Briseños (operan en el INFONAVIT las Brisas)-, las cuales básicamente están compuestas por adolescentes y comandadas por adultos.

Algunos jóvenes desertan de su familia, otros continúan viviendo con ella pero se aíslan de las actividades del hogar, no participan en la convivencia y no colaboran con las actividades que una unidad doméstica demanda. Dentro de la investigación realizada se observa que un $23 \%$ de los adolescentes declara que la comunicación que 
tienen con sus padres es mala o muy mala, y un $21 \%$ que la relación con toda su familia es mala o muy mala, al igual consideran que no existe la convivencia familiar. Muchas veces esto sucede no precisamente porque el adolescente así lo determine, sino porque las condiciones familiares así se le van dando:

En casa, todos trabajan, casi no nos vemos, mi papá el domingo todo el día está acostado viendo la tele, ese día mi mamá dice que ni toquemos al "jefe" 3 porque está descansando de toda la semana, ella, también trabaja, pero ese día se pone a lavar la ropa y limpiar la casa, mis hermanos cada quien sale por su lado y yo me quedó solo, me salgo de la casa; si mi mamá me ve sin hacer nada, me pone a trabajar.

Saúl 14 años, $2^{\circ}$ año de secundaria

Actualmente, la sociedad y las instituciones no son capaces de generar en todos los casos los espacios suficientes para los adolescentes. La familia no tiene una perspectiva de inclusión, al igual que la escuela, así que quienes no se ajustan a sus normas son rechazados o aislados; pocas escuelas realmente tienen programas para trabajar con adolescentes con problemas, las familias difícilmente pueden sobrellevar sus contradicciones cotidianas, su estabilidad es muy endeble, un "hijo rebelde" les cuesta trabajo poder atender, no saben cómo hacerlo y generalmente la disposición que muestran los padres es poca. Sin embargo, el no darles espacios a estos jóvenes en los centros educativos y espacios institucionales implica que aumente su posibilidad de caer en situaciones de riesgo.

El adolescente que desea convivir en el mundo socialmente aceptado por la mayoría, debe aprender a guardar sumisión ante la gente adulta (Ramos y otros, 2002), esto genera que los jóvenes entonces no se identifiquen siempre con sus valores sociales, así como con sus familiares. Los adolescentes que aceptan las reglas muchas veces no las hacen suyas, en ellos se observa la frase mexicana "Se obedece pero no se 
cumple", así realizan las cosas pero solamente por cumplir, pero no hay ni un cambio de conducta ni aprendizaje; en cuanto a la presión social se deja de ejercer, éste actúa como más le place, no asume con conciencia los mandatos sociales.

Sí limpio mi cuarto, pero a medias, nada más para que mi mamá vea que hago las cosas y no me esté molestando, pero ella misma dice que no hago bien las cosas, con la tarea igual, bajo las cosas de Internet y así las entrego, el maestro ni se da cuenta, hasta me felicita. Claro, hay unos teachers que sí desconfían pero no dicen nada, uno cumple y ya...

Raúl, 17 años, 3er semestre de bachillerato.

3- Hace referencia al padre de familia

\section{Medios}

Los medios de comunicación ocupan un amplio espacio en la vida de los adolescentes, absorben muchas horas de su tiempo, esto posibilita que los demás pierdan importancia y que aparentemente no sean tan necesarios. A pesar de que a los medios de comunicación se les ha acusado de absorber el tiempo de los niños y de los adolescentes, es importante señalar que de acuerdo con lo que mencionan los propios adolescentes, éstos ocupan el espacio que la familia o los propios amigos no les pueden dar.

Los adolescentes en México son el sector de población que más horas participa del tiempo libre, según el estudio realizado por el INEGI a través de la Encuesta 
Nacional sobre Uso del Tiempo (ENUT) 2002, en donde se observa que los hombres destinan un $17.3 \%$ y las mujeres un 17.0 de su tiempo (incluyendo las horas de sueño y descanso), esto implica que en promedio dedican más de cinco horas. En el estudio realizado por los autores de este texto se observan los siguientes datos (Ver cuadro 3) en cuanto al consumo de la TV por parte de los adolescentes.

Cuadro 3:

Tiempo diario que ven TV los adolescentes $\%$

1. No veo televisión

2. De 0 a media hora

3. De media a una hora

4. De 1 a 2 horas

5. De 2 a 3 horas

6. Más de 3 horas

SC

Total 100.0

Fuente: Encuesta Adolescencia sin otredad, 2005

La TV es el medio que más presencia tiene en sus vidas, por otro lado, de acuerdo con los resultados de este estudio, si sumamos los demás medios 4 de comunicación, radio, Internet, revistas, periódicos, cine, al igual que las nuevas tecnologías como los video juegos, teléfono celular (móvil), nos daremos cuenta de que les dedican más de cinco horas y media diarias promedio. Entonces, una tercera parte del tiempo que permanecen despiertos están frente a los medios de comunicación. 
Señalar que los medios de comunicación influyen o no en los adolescentes es caer en las posturas dicotómicas. Como lo señalan Orozco (1995) y Barbero (1995), hay un proceso de mediación, el cual es social e individual. Desde luego que los medios tienen un peso en la vida de ellos, son parte de su cotidianidad, los han incorporado a casi todas las actividades que ellos realizan, ellos mismos difícilmente perciben su vida sin medios de comunicación y sin nuevas tecnologías, éstos los acompañan a todas partes y son símbolos de jerarquía social. Gran parte de los adolescentes estudiados sí declaran necesitar y depender de ellos:

Sí, antes, cuando no tenía teléfono celular, no pasaba nada, pero ahora no puedo salir sin el mismo, si lo hago me siento incompleto... igual cuando en mi casa no está encendida la televisión se siente raro el ambiente, mi mamá dice que nada más gastamos luz (sic) porque la tenemos encendida y nadie la ve, pero si de repente se apaga, como que algo falta, se hace un silencio.

Elizabeth, $5^{\circ}$ semestre de bachillerato.

La televisión es, como se señalaba líneas arriba, la que tiene más presencia, sin embargo, también tienen importancia la Internet, la radio, los teléfonos celulares y los juegos de video. Ellos le permiten aislarse por momento de las relaciones sociales tradicionales, le facilitan establecer nuevos vínculos y que su entorno inmediato (familia, compañeros de escuela, vecinos) no sea indispensable para socializar.

Para algunos jóvenes los medios son indispensables para no depender de los demás, para aislarse, para desensibilizarse de su entorno, para enfrascarse en su propio mundo, desde luego esto en casos muy marcados no es una tendencia general, pero incluso son etapas que viven los adolescentes, en donde únicamente quieren estar relacionándose con las nuevas tecnologías y los medios de comunicación. Los medios 
de comunicación les permiten un vivir sin el otro, viven en el cine o en la televisión lo que no pueden vivir en su propia vida, ya que los "contenidos tocan la experiencia de la vida" (Covarrubias, 1994: 125), dan la apariencia que no necesitan de los demás para realizarse como seres humanos. Desde luego, esto es una etapa de vida que viven en cierto momento los adolescentes, no es una constante. Pero en el estudio se observa que en determinados momentos el mundo de ficción de los medios llega a tener muchas veces más peso para él que su propia vida real, por momentos los demás desaparecen, no existen los otros reales.

En este punto de la investigación, salta la duda de ¿Qué ocasiona que el adolescente se encuentre tan seducido por los medios de comunicación? ¿Qué provoca que ellos estén apegados más de la tercera parte de su día? ¿Cómo saber exactamente qué efectos tiene en ellos ante un espectro tan diverso de la juventud?

Los adolescentes visualizan a los medios de comunicación de diversas formas, por lo tanto, la percepción que tienen de los mismos no es una sola. Es importante señalar que existen identidades juveniles diferentes y divergentes (Brito, 2002), así que cada una de ellas responde de manera distinta a los mensajes de los medios de comunicación, no solamente hay diferentes interpretaciones (polisemia) del mensaje, sino también diferentes niveles cognoscitivos de analizarlo (multisemia); mientras unos tienen posturas críticas ante lo que sucede en los medios de comunicación, otros los ven de manera complaciente, aún en determinados momentos imitan de manera sistemática los mensajes de éstos. Como señala Orozco (1995), la propia identidad es una mediación en la medida que incide en la interacción que el sujeto tiene con los medios de comunicación. Sin olvidar que el mismo adolescente le da diferentes lecturas al mensaje y lo va resignificando a partir de las distintas experiencias de vida que tenga. 
Entonces, generalmente no tiene una mirada acrítica sobre lo que pasa en los medios, pues aunque muchas veces lo complacen no por ello deja de criticar su programación favorita. Los calificativos que tiene hacia los programas van desde que es un programa "tonto", "bobo", "estúpido" o hasta que es "muy bueno". Puede ser que el programa le parezca "menso", pero no por eso deja de verlo. Claro que no cree en todo lo que ve en la televisión, ni siquiera en los noticiarios televisivos que supuestamente dicen la verdad. Los medios los seducen, pero son audiencia activa (Lull, 1997)

La niñez va orientando la conducta del adolescente, aquellos que señalan que hubo una mayor presencia de la televisión y de los medios de comunicación como distracciones en su vida infantil, señalan que les cuesta más trabajo en la adolescencia establecer vínculos con sus padres y comprenderlos, lo cual se apega a lo que señala Hurlock (1995). No se puede generalizar respecto a esto, pero en las entrevistas y grupos focales realizados arrojan esa información. Aquellos que declaran que de niños convivían más con sus padres y menos con los medios, reconocen que en la actualidad hay rupturas y conflictos con los padres, pero que son poco frecuentes; y cuestiones como sexualidad, problemas en la escuela y de la vida en general son consultadas o comentadas con los padres, de preferencia con la mamá; pero aquellos que permanecieron más distantes de sus progenitores, no importando que vivieran juntos, utilizaban otras fuentes de información como los amigos y los medios de comunicación.

Desde luego, en las encuestas y en los grupos focales los sujetos investigados aceptan que los medios de comunicación, y de manera particular la televisión, les ha servido de referente durante su niñez y su adolescencia. 
Bueno, sí hay algunos chavos que se visten igual que los de la tele... mira, tengo amigas que quieren tener novios igualitos a Jorge Salinas, Eduardo Santamarina o ya de perdida el tal Yahir... es más, dicen que con ellos todo, sí, todo.. qué les pasa y son chavas que ya están grandes, tienen 16 años. Otras quieren vestirse igualito que la Britney o la tal Thalía, andan con sus ombligueras o sus tatuajes según ellas muy a la moda, pero nada, se les ve la panza por fuera, les digo que dan pena... este, en mi caso, pues no imito cosas de la tele... bueno algunas... es que cómo explicarte, no todo es malo, por ejemplo el otro día vi en una telenovela a Rubí, si el personaje principal, que la hace de mala que tiene un detallazo con el que iba a ser su esposo, y el otro se impresiona, bueno pues hice lo mismo con el que era mi novio y no... como que no le gustó, pero pocas veces hago eso de imitar lo que veo en la tele...

Marla, 17 años, estudiante de bachillerato.

Autores como Hurlock (1995) consideran que el adolescente obtiene sus modelos de imitación de los medios de comunicación, lo cual le genera conflictos porque las pautas de conductas proyectadas no son siempre aceptadas socialmente o bien son ridiculizadas. Lo cual es cierto en algunas circunstancias pero no se puede generalizar, el adolescente mediatiza; no hay un proceso directo; busca nuevos patrones que busquen romper con los valores que le habían mostrado en su casa, y si éstos se encuentran en los medios de comunicación intenta apropiárselos. "Me diferencio y luego existo" (Brito, 2002:50), el joven necesita diferenciarse de la mayoría, en varios aspectos, esto incluye los valores morales, llegando algunas veces a rechazar la moral convencional adulta empezado por los aspectos que están ligados a su infancia (Aguirre Baztán, 1998). Socialmente, entre más se diferencien los jóvenes más marcan su identidad, entre más se separen de las normas sociales establecidas más visibles se vuelven, por eso a veces los medios le sirven como referente para distinguirse de su entorno, sin embargo, no es una simple imitación. 
Desde luego que a veces sucede lo contrario, desean integrarse y ser aceptados por un espacio social y nuevamente utilizan a los medios de comunicación como referentes.

En este estudio también se observa la relación adolescencia-consumo-medios de comunicación, en donde estos últimos intentan persuadir a la población joven, tratando de generar estereotipos de consumidores, no obstante, no se puede decir que lo impongan, incluso la población joven ha generado movimientos que van en contra del consumismo como los movimientos contraculturales (Brito, 2002), los expertos en marketing saben que no todos los mensajes son eficaces y menos en este grupo etario (Otegui, 2004).

Es importante reconocer que la capacidad de consumo es un elemento importante en el joven adolescente, hasta autores como Brito (2002), señalan que el consumo genera identidades en la población joven, es un factor que permite distinguirse de otros e integrarse a un sector; también, de acuerdo con lo observado en esta investigación, puede incitar a conductas de riesgo. Esto último debido a que incluso hay adolescentes que rompen con sus valores tradicionales de grupo con tal de ver realizado su consumo, pudiéndose observar quienes se prostituyen para poder comprar aquellos productos que no son de primera necesidad y en su casa no se les pueden dar, otros optan por incorporarse a la delincuencia, pero es un sector juvenil mínimo, lo que sí es recurrente es el hecho de que hay adolescentes que se sienten frustrados por no poder consumir todo lo que la sociedad les ofrece, llegando a considerar culpables a sus padres o a todo su entorno. Un sentimiento de aislamiento lo invade por no sentirse digno de convivir con los demás y porque no le dan lo que considera debería tener. 
4- Por cuestiones de espacio no se presentan todos los resultados.

\section{Concluyendo}

La mayoría de los adolescentes estudiados no actúan de manera uniforme. Hay quienes en distintos momentos son asociales debido a que no tienen las competencias para adaptarse o convivir con un grupo, lo cual genera que muchas veces se les considere antisociales, generando que no desarrollen lazos afectivos con un grupo, la mayoría trata de ser sociales y algunos se esfuerzan, pues también es una cuestión de popularidad y de éxito (Hurlock,1999). Sin embargo, por momentos el adolescente desea estar sin los otros y esto no solamente hace referencia a lo físico, sino también a lo emocional y axiológico.

El adolescente viene desarrollando una vida en donde la presencia adulta desde su niñez es poca, y cuando existe es para sancionar y vigilar, no para la convivencia, al menos en la mayoría de los casos observados. El tener novi@ y amig@s por momentos se vuelve una necesidad, aunque los adolescentes en la medida que idealizan este tipo de relaciones generalmente salen decepcionados, y muchas veces se involucran en dinámicas de dependencia, por lo tanto, cuando superan este tipo de relaciones consideran que la convivencia con los demás no es garantía de sentirse mejor. A los adolescentes les cuesta trabajo reconocer el esfuerzo que hace el entorno por ellos, de manera particular sus padres, amigos, escuela y otras instituciones, esa desvalorización los hace sentirse solos y les genera resentimiento. Por otro lado, consideran que los adultos en general, y muchas veces todo su contexto, solamente ven sus defectos, y ven a los otros como sus enemigos. 
La relación con los padres es compleja, tratan de independizarse de ellos, han aprendido a estar sin su presencia, pero a la vez saben que dependen de éstos y declaran que los aman profundamente, reconocen que son ellos quienes los sacan de cualquier apuro; sin embargo, no soportan su compañía por mucho tiempo, ni pueden llegar a considerarlos sus cómplices. La adolescencia sin otredad es una etapa de vida que llegan a vivir algunos adolescentes en la actualidad, los cuales desde niños han tenido que vivir con poca presencia adulta; por momentos consideran que los demás en su vida no tienen importancia, los piensan diferentes a sí mismos, se sienten solos y hasta prefieren estar aislados, no se sienten parte de una identidad colectiva, aunque por momentos deseen serlo construyéndose un sentimiento encontrado; se consideran dañados emocionalmente por los demás (padres, amigos, escuela, y demás miembros del entorno), en donde los medios de comunicación y las nuevas tecnologías tienen una gran presencia, incluso se les facilita más iniciar y mantener relaciones a través de estos medios y éstos se convierten en referentes en la construcción de su identidad, desde luego a través de un proceso de mediación. Este estado puede llegar a producir conductas de riesgo: adicciones, depresión y dependencia emocional, con las respectivas consecuencias de cada uno de estos estados.

\section{Fuentes de consulta}

Aguirre Baztán, Ángel (1998). Psicología de la adolescencia, Alfa Omega Marcombo, España.

Barra Ruatta, Abelardo (2000) Filosofía de la otredad. Educar para la diferencia. Revista Contextos de Educación No. IV. Argentina, Universidad Nacional de Río Cuarto. Disponible en http:/ / www.unrc.edu.ar/publicar/cde/Barra\%20Ruatta.htm 
Brito Lemus, Roberto (2002). Identidades juveniles y praxis divergente; acerca de la conceptualización de juventud. (Nateras Domínguez, Alfredo; Coordinador) Jóvenes, culturas e identidades urbanas, Universidad Autónoma de México, Miguel Ángel Porrúa, México, D.F.

CEPAL (2002) Elevadas tasas de deserción escolar en América Latina. Informe de la CEPAL Panorama Social de América Latina 2001-2002. Comisión económica para América Latina y El Caribe, Naciones Unidas. Consultado: Febrero 2005) Disponible en: http://www.eclac.cl/cgibin/getProd.asp?xml=/prensa/noticias/comunicados/0/11260/P11260.xml\&xsl=/ prensa/tpl/p6f.xsl

CONADIC, (2003) Encuesta nacional de adicciones. Tabaco, Alcohol y otras drogas, Consejo Nacional de Adicciones, México.

Conger, John, Adolescencia (1999). Generación presionada, Harla, México

Covarrubias Cuellar, Karla Yolanda y otras (1994)

Cuéntame en qué se quedó / Trillas, FELAFAC, México.

Flores, Ociel (1999) “Octavio Paz. La otredad, el amor y la poesía”, Revista Razón y palabra, Número 15, Año 4, Agosto - Octubre 1999, Mëxico, ITESM.

Disponible en http://www.cem.itesm.mx/dacs/publicaciones/logos/anteriores/n15/oflores15.ht $\mathrm{ml}$ 
Gall, Olivia (2004) Identidad, exclusión y racismo: reflexiones teóricas y sobre México. REVISTA MEXICANA DE SOCIOLOGÍA, AÑO 66, NÚM. 2, ABRIL-JUNIO, 2004. Disponible en http://www.ejournal.unam.mx/rms/2004-2/RMS04201.pdf

Herrera, Óscar (2005). Reclutan criminales a niños de 14 años, 19 de abril 2005, El Universal, primera sección, México.

Gabelas, José Antonio y Marta Lazo, Carmen (2008) Consumos y mediaciones de familias y Pantallas. Gobierno de Aragón, Zaragoza.

Guadarrama Luis Alfonso, Valero Jannet y Brito Karla (2004). El top ten de la sexualidad saludable, ISEM, UAEM, FAMECOM, México.

Hurlock, Elizabeth B. (1999). Psicología de la adolescencia / -- 11. ED. -- México: Paidós

INEGI 2002. En busca del tiempo perdido, Fundación este País, México. Revista Este País. Disponible en:http://www.consulta.com.mx/interiores/99_pdfs/15_otros_pdf/oe_2002_UsoTi empoEstePais.pdf

Lull, James (1997) Medios, comunicación, cultura: aproximación global Argentina : Amorrortu.

Marta Lazo, Carmen (2005) La televisión en la mirada de los niños, Editorial Fragua, España.

Marta Lazo, Carmen (2005) "Agentes mediadores y responsables del consumo infantil de televisión: familia, escuela y medios de comunicación", Revista 
Comunicación y hombre: revista interdisciplinar de ciencias de la comunicación y humanidades, Universidad Francisco de Vitoria, Madrid, España, № . 1, 2005, págs. 19-34.

Marta Lazo, Carmen y Gabelas Barroso, José Antonio (2007) La educación para el consumo de pantallas, como praxis holística, Revista Latina de comunicación social, $\mathrm{N}^{0}$. 62, Universidad de La Laguna, Tenerife, España.

Madrigal Guadalupe (2004). Sin consenso, reforma para secundaria: Tamez, Noticieros Televisa, Nota con fecha 14 Julio de 2004 (Consultada en septiembre de 2005) Disponible en: http://www.esmas.com/noticierostelevisa/mexico/377630.html

Moreno Kena (Coordinadora)(2003) Drogas: las preguntas más frecuentes. Centros de Integración Juvenil. México.

Montero Maritza (2001) Ética y política en Psicología. Las dimensiones no reconocidas, Athenea Digital - num. 0 abril 2001, España, Universidad Autónoma de Barcelona

http://antalya.uab.es/athenea/num0/maritza.htm

Ramos Cavazos Ma. Teresa y Cantú Martínez Pedro C. (2003). EL VIH/SIDA y la adolescencia, Revista de la Facultad de Salud Pública y Nutrición, Universidad Autónoma de Nuevo León (México) Vol. 4 No.4 Octubre-Diciembre 2003.

Obregón, Rafael (2001). Medios y salud pública: La voz de los adolescentes. Proyecto Comsalud, OPS y OMS, USA. Los Colaboradores de este estudio fueron: 
Dra. Gloria Coe, quien en ese momento era Asesora Regional, OPS, Washington, DC; Matilde Maddaleno, Directora de Programas de Salud de Adolescente, OPS, Washington, D.C., los(as) profesores(as) Rina Alcalay, Universidad de California, Davis; Luis Alfonso Guadarrama Rico, Universidad Autónoma del Estado de Mexico, México; Ana María Cano, Universidad de Lima; Jair Vega, Universidad del Norte, Paulo Lyra, Asesor, Comunicación y VIH/SIDA, OPS, Washington, D.C., y de los investigadores principales designados por 13 facultades de comunicación social de América Latina para atender el Taller de Revisión del Protocolo celebrado en Ibarra, Ecuador del 1-6 de Julio, 2001.

Otero-López, José Manuel (1997) Droga y delincuencia, Un acercamiento a la realidad, Psicología Pirámide, Madrid.

Otegui, J.M. (2004). La influencia de los medios de comunicación en la transmisión de valores. ón de valores. En Los medios de comunicación y el consumo de drogas. Avances en Drogo dependencia (Coordinadores Pantoja y Abeijón) Instituto de Deusto de Drogodependencias. Universidad de Deusto, Bilbao.

SEP (2004). Comunicado encuesta de la OCDE, Febrero de 2004, SEP, Disponible en:

http://www.sep.gob.mx/wb2/sep/sep_comunicado070204

Velasco Fernández, Rafael (2001) La familia ante las drogas, Trillas, México

Velasco Fernández, Rafael (2003) Las adicciones. Manual para maestros y padres, Trillas, México 